\title{
Preventing cold chain failure: vaccine storage and handling
}

Background and epidemiology:

Most of the currently available publicly funded vaccines in Canada (Box 1) must be stored between $2^{\circ} \mathrm{C}$ and $8^{\circ} \mathrm{C}$ to maintain their potency. The "cold chain" refers to the continuum $b$ of safe handling practices, including materials, equipment and procedures, that maintain vaccines within this temperature range from the time they are manufactured to the time they are administered to patients. Physicians are a vital clasp in the

Box 1: Publicly funded vaccines and testing solution that must be stored between $2^{\circ} \mathrm{C}$ and $8^{\circ} \mathrm{C}$

- Diphtheria and tetanus toxoids

- Diphtheria and tetanus toxoids, and pertussis vaccine (acellular)

- Diphtheria and tetanus toxoids, adsorbed pertussis vaccine (acellular) and inactivated polio vaccine

- Diphtheria and tetanus toxoids, pertussis vaccine (acellular), inactivated polio vaccine and Haemophilus influenzae type b (Hib) conjugate vaccine

- Hepatitis A vaccine

- Hepatitis B vaccine

- Hib conjugate vaccine

- Inactivated polio vaccine

- Influenza vaccine

- Measles, mumps and rubella vaccine*

- Meningococcal C vaccine

- Pneumococcal 7-valent conjugate vaccine

- Pneumococcal polysaccharide 23-valent vaccine

- Rabies vaccine

- Rabies immune globulin

- Tuberculosis testing solution

- Varicella vaccine*

*The diluents for these vaccines do not have to be stored in the refrigerator. chain, which too often is broken. The most common reasons for spoilage are refrigerators that are either too hot or too cold for safe vaccine storage, and vaccines that are left out of the refrigerator for too long.

According to Ontario data, up to $20 \%$ of physician offices or health care facilities do not meet the necessary requirement of vaccine storage and handling. As well, about $4 \%$ of all vaccines ordered by doctors' offices and clinics expire before they are used. The estimated cost of wasted vaccines in Ontario is more than $\$ 3$ million annually. This amount is likely an underestimate because it is based only on the wastage reported or returned to the ministry; the amount of vaccine that is discarded in doctors' offices or administered with suboptimal potency because of breaks in the cold chain is unknown.

Clinical management: Vaccines should be stored in a refrigerator dedicated for this purpose. Lunches and specimens should be stored elsewhere. Using a maximum-minimum thermometer is the only way to know if the fridge is maintaining the right temperature range, between $2^{\circ} \mathrm{C}$ and $8^{\circ} \mathrm{C}$. These thermometers - available in digital and mercury - are designed to indicate the minimum and maximum temperatures inside the fridge since the thermometer was last reset. These temperatures should be recorded and the thermometer reset daily.

Never store vaccines on refrigerator-door shelves, where they are exposed to warm air every time the door opens. Always store vaccines on the middle shelves of the fridge and allow sufficient space for air to circulate around them. Placing water bottles on the bottom, top and door shelves helps maintain temperature consistency. Installing an inexpensive latch ensures that the fridge door closes properly each time. More than $1 \mathrm{~cm}$ of ice in the freezer compartment signals the need to defrost the fridge. During this process, transfer the vaccines to an insulated vaccine carry bag or another fridge set at the correct temperature range and check the temperature regularly. It goes without saying that vaccines must be returned to the fridge immediately after use.

To minimize the risk of expiration, order only the amount of vaccine that can be used in 1- to 3 -month periods. Place vaccines of the same type together. Check expiry dates regularly and move older vaccines to the front of the fridge and use these lots first. Mark the date when the first dose is withdrawn on all multidose vials, and discard opened vials within 30 days, unless otherwise specified by the manufacturer.

Prevention: Public health staff should conduct regular onsite inspections of the cold-chain management practices at clinics and health care facilities to provide education and ensure safe handling of vaccines. Most local public health units have a vaccinepreventable disease team available to assist when problems with temperature maintenance, power loss and possible spoilage arise.

\section{Erica Weir}

Associate Medical Officer of Health Kathy Hatch

Infectious Disease Prevention Program

Kingston, Frontenac and Lennox \& Addington Health Unit

Kingston, Ont. 